\title{
SECURITY PROBLEMS OF COMMUNICATIVE STRATEGIES
}

\author{
Aleksander I. Dontsov \\ Lomonosov Moscow State University \\ Moscow \\ Elena B. Perelygina \\ Humanitarian University \\ Ekaterinburg
}

\begin{abstract}
The main directions of safety formation in communication strategies are connected with their adaptation to the conditions of social and psychological variability. Building up a communicative strategy is a versatile process, in which it is necessary to consider a wide spectrum of social and psychological parameters, especially topical in the modern period of social development. Forms of social interactions in schematic formats of contemporary social and economic revolution are reduced, social and functional potentials are depleted, mass society is further differentiating, the pace of historic changes is growing, all this determines the necessity to prepare changes in personality structures to the dynamics of social and psychological fluidity.
\end{abstract}

Keywords: co-presence, compatibility, dialogue.

J.Baudrillard pointed out that in contemporary world the $I$ - The Other interaction strategy undergoes depersonalization. One of the most important and alarming phenomena of Russian communicative culture - which not only prevents economic multicultural communication but also is a basis to social and organizational deformations - is an underdeveloped notion of The Other in the national culture.

Communicative effect management, consumer behaviour management... Dreams about manageable world face the reality of arrogance towards individuality, pococuranteism to the right of The Other to be different, disregard to the targets of compatibility, contextual interaction.

Not occasionally new tones are found in the concept of A. Giddens, which reveals situational contexts of presence and co-presence in 
social interaction. In his interpretation the structural properties of social systems reflect "the dialectics of presence and absence in time and space", which connects the simplest forms of social action with structural properties of the society. According to Giddens, when the conditions of co-presence change from the point of view of social and psychological aspects (compatibility, mediated social interaction), the relations with those physically absent require different social mechanisms. Thus, the absent others, who at any moment can intrude by means of a mobile phone into the time and space of a mobile phone user, create premises for re-estimation of compatibility, the private and the public, the far and the close. Thereby, owning a small portable means of distant communication creates conditions for new complex forms of social interaction both with the present and the absent others.

In the situation of co-presence mutual interaction depends on basic social and psychological parameters of interdependence. A high level of dependence (and interdependence) is often connected with subjective feeling of dependence and with focus on long-term relationships (i.e. with a degree of commitment to them). This kind of involvement is a powerful determinant of pro-social behaviour in the context of personal connections, as well as formal organizations; while the growth of independence, on the contrary, is often related to the decrease of pro-social behaviour. For example, availability of good alternatives, which reduce the dependence, is more often connected with a stronger focus on personal achievements. When the dependence is high the possibilities of exploitation from the side of one or both partners are low. Such interactions and relationships are more stable and imply mutual cooperation. If the level of mutuality in the dependence is low, the exploiting potential is much higher, what is usually connected with lower stability in relations and less pronounced cooperation.

It is symptomatic what the head of international institute specializing in language, communication skills, and cross-cultural training $\mathrm{R}$. Lewis advises on business communications with Russian partners: "dissidence... is in general not popular with Russians. Security has historically been found in group, conformist behaviour. Do not try to separate a Russian from his or her 'group' whatever that may be".

Dependence and coherence of interaction are understood in a different aspect by David Deutsch, who specified interdependence groups 
of three types (cooperative, mixed motivation, competitive) and pointed out that the group type influences on communicative potential. These researches show that the cooperative type has less communicative threats, consistency of actions and mutual attraction are higher in such groups in contrast to competitive groups, which have more communicative contradictions. In the mixed motivation groups, characterized by a combination of cooperative and competitive elements, there is a social dilemma, in which an individual member is better to produce as little as possible, but in the interests of the group, on the contrary, it is best when everyone contributes to the common objective (van Knippenberg, Wilke). Thus, most pro-communicative social interaction is inherent to groups with cooperative interdependence.

Among other fundamental factors in the strategic communication formats devotion, which corresponds to man's desire to maintain relationships and to feel psychologically subordinate to these relationships, as well as social support, which has an overall positive effect on psychological well-being, can be emphasized (Buunk, Bierhoff).

Thus, security of communications depends a lot on the level of acts coordination among the participants of interaction, as well as on the degree of commitment and social support which contribute to the psychological stability of the social interaction participants.

In the new book "Security and Organization", published in Milan, Cesare Kaneklin points: "While the number of regulations and general references of European states to security issues increases, a counterdesire for personalization and identification of common orientations of modern communicative security grows daily" (Sicurezza).

Although participants are able to track most of what is happening in a broader range of social environment, focused interaction clearly separates those who participate in it, and those who are co-present. $A$ unit of focused interaction is a face to face meeting or an encounter that is a road map of social interaction, the continuity of meetings with each other, well-ordered in the everyday cycle of activities. "That is, what from the angle of the fleeting moment might appear brief and trivial interchanges take on much more substance when seen as inherent in the iterative nature of social life. The routinization of encounters is of major significance in binding the fleeting encounter to social reproduction" (Giddens). Optional requirement of compatibility and participation, conformity to the 
methods of co-presence brings into the regulation of social behavior the phenomenon of "norms absence", a continuing situation of anomie.

Relationships of trust are of no less significance for the expansion of space-time distance in social interaction, for communicative efficiency, and for the implementation of systematic integration. Emphasis on the predominance of tact, recovery from the deformation of social structure and maintaining trust in social interactions presupposes primary attention to the protection of social integrity, internal mechanisms of social reproduction, strengthened dialogue with the Others.

Today, according to many researchers, there is a crisis of communication that in the interpretation of J. Baudrillard, is due to imitation of the dialogue process. He notes that "the entire system of communication has moved from the complex syntactic structure of language to a binary system of question / answer signals or the system of continuous testing. Yet we know that the test and the referendum are the ideal forms of simulation: the answer is suggested or pre-modeled / designated by the question.... In other words, the content of communicative interaction is determined by a greeting message, as well as its informative and vector modeling is determined by the initiative act of communication.

Other aspects of communicative crisis are no less important. Advisory Board Chairman of the Political Philosophy Center A. Kolesnikov together with his colleagues reveals communicative backwardness of our country and the lack of communicative strategies, based not only on insufficient quantity and quality of infrastructure: "That is the crisis of communications, communicative retardation. Hence, during and after the crisis endeavours to improve the quality of communication, to change the discourse - the style and content of conversation with the nation and the world - will be of vital importance". For example, to be in a Group of Twenty and to talk to it in the language of confrontation is impossible because it is economically and politically costly. To deprive people of a wide menu of political participation means to underestimate the citizens, who are already feeling the negative consumer and social consequences of the crisis, and therefore they need a dialogue with the authorities, the real influence on decision-making, working feedback mechanisms (elections, mass media, civil organizations). Overcoming the communicative crisis means overcoming peripheral character of Russia as a state (Dragunskiy, Zharkov, Kolesnikov). 
In this regard, an important part in our research is to appeal to such a social and psychological parameter, as inhibition, which is of particular importance in conservation strategies for communication security. Inhibition, described by George Herbert Mead, is the containment of impulses by the participants of interaction, the ability to act selectively, and is an essential component of secure social interaction. The culture of communicative interaction in the complex and dynamic society is a limiting factor for the emergence of complex and crisis situations that are so abundant in modern society. And these situations are volatile, durable, and historically divergent. Ch. Cooley wrote: "The substitution of higher forms of control for lower, the offering of more alternatives and trusting the mind to make the right selection, involves, of course, an increased moral strain upon individuals. Now this increase of moral strain is not in all cases exactly proportioned to the ability to bear it well; and when it is not well borne the effect upon character is more or less destructive, so that something in the way of degeneracy results". When there is an emphasis on the aims of success in the culture, conformity to institutionally prescribed formats and strategies for achieving this goal weakens, but the existence of samples of increasing optimism associated with the chances for success in the professional sphere is very important.

In modern society communication strategies and social and psychological parameters undergo a change. Social sphere moved to a new level of co-presence and compatibility, which is formed by a complex interacting mass of organizations, groups and relationships. Thus, in the U.S. now about 1 million 370 thousand companies interact with more than 90 thousand of schools and universities, 330 thousand of churches and hundreds of thousands of branches, 13 thousand of national organizations, plus countless environmental, social, religious, sports, political, ethnic and civic groups, each with their own values and priorities. The country has about 144 thousand law firms, providing services to all of these interactions (Toffler). In addition, significant changes occurred in the information sphere. Balanced communication between social groups and institutions are provided by global increase in news reports, links, and flows. The challenges in communication strategies for inhibiting, preventing differentiation of values and aggressive motivations are recognized. 
The results of the undertaken study ${ }^{1}$ show that the greatest concern is the threat of mass manipulation ( $75 \%$ of respondents), while the interpersonal manipulation concerns only $20 \%$. The respondents stated that the situations of watching TV cause the greatest number of fears and concerns regarding the content of the information received (38\% of respondents). In terms of interpersonal communication the greatest concern is the lack of inhibition in professional and business sphere $42 \%$, in the sphere of family and relatives $-21 \%$, and in the sphere of friendship $-14 \%$. Thus, the issue of individual security depends on the impact of mass media and the use of numerous manipulative techniques that, intruding into the inner world of a person, destroy his/her usual stereotypes, values and distort the personal worldview.

Granted the rejection of the limitations upon behavioural capabilities associated with a detached individual, it is possible speak about a program, which means by itself a strategy. Basing on Luhmann's position, it is advisable to consider the program as a set of conditions for socially acceptable behaviour that are to ensure a safe field of communication. At the same time the programs of strategic level should be formulated systematically, with the possibility of correction and without detailed differentiation. In this case, the axiological consensus facilitates the communication about the contingency of programs, that is the development of programs, adapting them to the situation, their change or aging (Luhmann).

Furthermore, in the opinion of E. Mounier, it is important to follow a number of interactive provisions in the strategies of communicative interaction. First, one needs to go beyond the limits of their self - to separate from oneself, to reveal oneself, to disclose to the Other. Second, it is important to go beyond one's own point of view, and be able to adopt the position of the Other, that is to combine different positions in reaching an agreement. Third, one is to be capable of taking responsibility - taking responsibility for the destiny of the Other. Four, one is to be able to give generously and freely. "Generosity, even if it does not receive a response, melts the ice of distrust and breaks the circle of soli-

\footnotetext{
${ }^{1}$ The study involved 176 respondents (90 women and 86 men) aged 20 to 64 years, students of Humanities University (Ekaterinburg), workers in the banking sector and the police.
} 
tude, smoothens the rough edges of disagreement" (Mounier). And finally, one is to be loyal - "loyalty, love and friendship are perfect, only if they are constant. Constancy has nothing to do with resistance, nor with uniformity, it is a relentless emanation" (Ibid.).

Social and psychological parameters of social interaction largely depend on the type of culture, as well as on certain behavioural strategies related to national and mental traits. For example, according to writers British people are characterized by: ethics and activity in overcoming the obstacles, the ability to respond to life situations in an appropriate manner (the cult of prescribed behaviour) during interpersonal and social communication, regulation of emotional expressiveness, self-restraint and courtesy, the need for communication, communicative sensitivity. Keeping the "stiff upper lip" for an Englishman is mediated by moral norms and the opinion of others about his behaviour. At the same time the rejection of the impact on other people's settings and views, benevolence, politeness in communication, avoidance of psychological pressure are inherent in British people (Sukharev, Sukharev).

A well-known Russian journalist and writer V. Ovchinnikov, exploring the national character of different nations, concludes that the development of such traits in the British and, above all, of their correctness, grows out of an instinct of social self-preservation. Prevalence in the British character of repressiveness, i.e. self-control, self-command, especially in crisis situations, the cult of prescribed behaviour comes from the benefits of communicative security.

Substantial difference in cross-cultural patterns of interaction necessitates considering the differences of social and psychological parameters and factors in intercultural relations. It is known that the Japanese forms of personal pronouns to refer to the speaker or the one to whom the speech was addressed, were varying endlessly according to strict rules of etiquette, depending on the rank of each communicator and what were their associated social relations. When the number of social classes decreased dramatically, and then they disappeared completely, it became very difficult to know how to treat a person in order to avoid complicated or threatening communicative situations. In doubtful cases, instead of "I" or "you" people began to apply more and more vague impersonal forms, preferring neutral "They believe that ..." instead of "I think that", 
not to make an accidental mistake by using a situationally improper personal pronoun (Frederick).

A tendency to create total information space with the mandatory coding of social interaction subjects removes the significance of rituals and etiquette of compatibility, moral and value regulation, creates a "hallucinatory" freedom of communication.

In conclusion, we can use an anti-simulation humanistic thesis of Ch. Cooley: "In so far as [the society] favours, in every child, the development of his highest possibilities, it is free, but where it falls short of this it is not. So far as children are ill-nurtured or ill-taught, as family training is bad, the schools inefficient, the local government ill-administered, public libraries lacking $\langle\ldots\rangle$ in so far the people are unfree". 